\title{
Vorpolitische Grundlagen des demokratischen Verfassungs- staates bei Jürgen Habermas und Joseph Ratzinger
}

Vorpolitische Grundlagen des demokratischen Verfassungsstaates werden zumeist im Zusammenhang mit dem Böckenförde-Paradoxon diskutiert. Nach Ernst-Wolfgang Böckenförde lebt der freiheitliche, säkularisierte Staat von Voraussetzungen, die er selbst nicht garantieren kann. Darin drückt sich der Zweifel aus, ob der demokratische Verfassungsstaat seine normativen Bestandsvoraussetzungen aus eigenen Ressourcen erneuern könne, sowie die Vermutung, dass er auf weltanschauliche bzw. religiöse, jedenfalls kollektiv verbindliche ethische Überlieferungen angewiesen sei. Im Januar 2004 führten zwei Antipoden - Jürgen Habermas, der nachmetaphysische Philosoph, und Joseph Ratzinger, zu jenem Zeitpunkt Kardinal und Präfekt der Glaubenskongregation in Rom - einen Dialog zu diesem Streitthema. Ausgehend von diametralen Grundpositionen kamen beide Protagonisten wider Erwarten zu gleichen Schlussfolgerungen, zu der Notwendigkeit eines wechselseitigen Wahrnehmungs- und Lernprozesses. Allerdings zeigen beide Ansätze aber auch deutliche Schwächen hinsichtlich ihrer Realisierbarkeit: Habermas müsste von seiner Außenperspektive zur Religion abrücken, um den Gehalt der Religion in seiner Bedeutung für die Verankerung von Moral vollständig zu erfassen, während es Ratzinger kaum gelingen kann, seinen Ansatz in einer pluralistischen und in weiten Teilen schon säkularen Gesellschaft durchzusetzen.

Inhalt

1. Einleitung $\quad 380$

2. Vorpolitische Grundlagen politischer Ordnung 380

3. Argumentationslinien der Dialogpartner 382

a) Jürgen Habermas $\quad 382$

b) Joseph Ratzinger 384

4. Vergleich beider Ansätze $\quad 386$

5. Kritik 388

$\begin{array}{ll}\text { Literatur } & 390\end{array}$

Zeitschrift für Politikwissenschaft 17. Jg. (2007) Heft 2, S. 379-392 
Ines-Jacqueline Werkner

\section{Einleitung}

„Es heißt die Einheit der Welt verkennen, wenn man Religion und Politik für grundverschiedene Dinge hält, die nichts miteinander zu schaffen hätten noch haben dürften [...]. In Wahrheit tauschen sie das Gewand, [...] und das Weltganze ist es, das redet, wenn eines des anderen Sprache spricht.“ (Mann 2004: 107) - so stellen sich für Thomas Mann im vierten Teil seiner Tetralogie „Joseph und seine Brüder“ Religion und Politik als Ausdruck ein und derselben Wirklichkeit dar.

Bei der Verhältnisbestimmung von Religion und Politik scheint es sich - das zeigen bereits Überlegungen von Augustinus zu den beiden „,civitates“ oder die sich daran anschließende Zwei-Reiche-Lehre der Reformation - um eine fortwährende und dem Christentum immanente Debatte zu handeln. Letztlich geht es bei der Frage nach Inklusion oder Exklusion von Religion im politischen Diskurs vor allem um die Struktur der Begründungslogik öffentlicher Vernunft. Dabei zeigt sich, dass sich das neuzeitliche Bewusstsein an diesem Punkt keineswegs im Einklang mit sich selbst befindet, sondern Spannungen enthält, die dem liberalen Projekt selbst eingeschrieben sind. Ausdruck dafür ist die auf Ernst-Wolfgang Böckenförde zurückgehende Frage nach den substanziellen Bestandsvoraussetzungen moderner Gesellschaften. Wenn es denn zutreffen sollte, dass der freiheitliche, säkularisierte Staat von Voraussetzungen lebt, die er selbst nicht garantieren kann (Böckenförde 2006: 112), stellt sich die Frage, ob die Begründungslogik öffentlicher Vernunft ihrerseits in dieses Angewiesenheitsverhältnis einbezogen ist oder nicht (Seibert 2006).

Im Mittelpunkt des Beitrages steht der 2004 genau zu diesem Streitthema geführte Dialog zwischen Jürgen Habermas, dem nachmetaphysischen Philosophen, und Joseph Ratzinger, zu jenem Zeitpunkt Kardinal und Präfekt der Glaubenskongregation in Rom. Beide Protagonisten diskutierten über vorpolitische Grundlagen des demokratischen Rechtsstaates, über das, was politische Gemeinschaften in ihrem Innern zusammenhält. Dabei zeigen beide Ansätze - so meine These - deutliche Schwächen hinsichtlich ihrer Realisierbarkeit: Habermas müsste von seiner Außenperspektive zur Religion abrücken, um den Gehalt der Religion in seiner Bedeutung für die Verankerung von Moral vollständig zu erfassen, während es Ratzinger kaum gelingen kann, seinen Ansatz in einer pluralistischen und in weiten Teilen schon säkularen Gesellschaft durchzusetzen.

Ausgehend von kurzen Anmerkungen zum Begriff der vorpolitischen Grundlagen werden zunächst die Hauptargumentationslinien von Habermas und Ratzinger dargestellt. In einem weiteren Schritt werden beide Positionen miteinander verglichen, um dann abschließend in kritischer Analyse beider Ansätze die obige These zu begründen.

\section{Vorpolitische Grundlagen politischer Ordnung}

Die Frage nach vor- bzw. außerkonstitutionellen Bedingungen politischer Ordnung ist in der neueren politischen Theorie - so Herfried Münkler (1996: 7) - bisher eher vernachlässigt worden. Vieles blieb ausgespart, weil es nicht rationalisierbar oder institutionalisierbar war oder weil es durch die Institutionalisierung selbst destruiert wurde. Dies betraf vor allem die sozio-moralischen Grundlagen der politischen, sozialen und wirtschaftlichen 
Ordnung (Münkler 1996: 7). Sie gelten als vor-politisch, insofern sie Voraussetzung politischer Ordnung sind, ohne selbst Bestandteil des politischen Systems werden zu können und in dessen Kreisläufe der Selbstreproduktion integriert zu sein. Damit gehören sie sozusagen zur politischen Verfasstheit, nicht aber zur politischen Verfassung (Münkler 1996: 8).

Der Status des Vorpolitischen resultiert dabei aus der funktionalen Ausdifferenzierung der Gesellschaft. War in früheren Epochen der Geschichte das Politische und Sozio-Moralische bzw. Religiöse eng miteinander verbunden, erfolgte in der Neuzeit die Trennung von Staat und Kirche und das Herauslösen religiöser und sozio-moralischer Faktoren aus dem Bereich des politisch Verfügbaren. Mit diesem Prozess der Entpolitisierung und Privatisierung des Religiösen und der moralischen Dispositionen der Bürger gingen zwei Entwicklungslinien einher: Zum einen war damit eine Erweiterung der individuellen Freiheit und Selbstbestimmung verbunden. Insofern wurde dies als politischer Fortschritt begriffen. Zum anderen entstand daraus aber auch gleichzeitig die Gefahr selbstdestruktiver Konsequenzen dieses Fortschritts, insofern er ein Verhalten begünstigte, zugunsten größtmöglicher Freiheit deren Voraussetzungen und Bestandsbedingungen zu vernachlässigen (Münkler 1996: 9).

Während beispielsweise Münkler $(1993,1994)$ diese Problematik am Gegenstand der Bürgertugend in der Dimension von Liberalismus und Republikanismus thematisiert, findet die Diskussion zwischen Habermas und Ratzinger um vorpolitische Grundlagen und das rechte Verhältnis von säkularem Verfassungsstaat und Religion dagegen eher im Spannungsfeld von Politik, Recht und Moral in der Dimension von Naturrecht und Rechtspositivismus statt.

Dem Naturrecht liegt die wesentliche Überzeugung zugrunde, dass jeder Mensch „,von Natur aus“ mit unveräußerlichen Rechten ausgestattet sei. Dabei wird versucht, „faktisch bestehendes Recht in Richtung auf eine Ordnung zu transzendieren, die menschlicher Setzung bzw. Vereinbarung vorausliegt und diese normiert.“ (Forschner 2002: 186) Die Normen gelten, weil sie ,,aus Gott, der Natur oder Vernunft stammend, gut, richtig, gerecht sind“ (Kelsen 1928: 8). Auf diese Weise werden - im Gegensatz zum strengen Rechtspositivismus - moralische Elemente in den Rechtsbegriff implementiert (Alexy 1990: 10).

Im Gegensatz zu naturrechtlichen Denkformen, die zur Nivellierung der Differenz von Recht und Moral neigen, betont der Rechtspositivismus dagegen die begriffliche Trennung von Recht und Moral, das heißt: „Ob eine bestimmte Norm oder Normenordnung Recht ist, hängt von Kriterien ab, die sämtlich außermoralischer Natur sind.“ (Hoerster 1990: 27) Die einzigen Voraussetzungen des Rechtsbegriffs bilden in formaler Hinsicht die ordnungsgemäße Satzung und die soziale Wirksamkeit, während material gesehen - so in der Auffassung von Kelsen - ,jeder beliebige Inhalt Recht sein [kann]“ (Kelsen 1960: 201). Grundlage der Rechtsgeltung ist damit nicht mehr die Anerkennung eines überpositiven Maßstabes der Menschenwürde oder des Naturrechts, sondern allein die Verfassung der jeweiligen Staaten. Habermas nimmt die Argumente dieser beiden Pole - Naturrecht versus Rechtspositivismus - auf und versucht, mittels eines integrativen Ansatzes die jeweiligen Schwächen beider Positionen auszugleichen. Mit seinem diskursethischen Rechtskonzept steuert er „Zwischen den Klippen des Rechtspositivismus und des Naturrechts hindurch“ 
Ines-Jacqueline Werkner

(Habermas 1998c: 668). Ratzinger vertritt dagegen einen naturrechtlichen Ansatz mit Gott als Ursprung und Quelle von Vernunft.

\section{Argumentationslinien der Dialogpartner}

\section{a) Jürgen Habermas}

Dem Dialog dieser beiden Protagonisten ging Habermas' Friedenspreisrede im Jahr 2001 voraus. Seine von der Öffentlichkeit mit Erstaunen aufgenommene Hinwendung zur Religion wurde als „,Steilvorlage“ für die Kirchen“ (Schuller 2005: 10) bezeichnet und kann auch als Ausgangspunkt des hier thematisierten Gesprächs gelten. Im Fokus der damaligen Rede stand der Begriff der ,postsäkularen Gesellschaft, die sich auf das Fortbestehen religiöser Gemeinschaften in einer sich fortwährend säkularisierenden Umgebung einstellt" (Habermas 2001: 13).

Im Dialog mit Joseph Ratzinger knüpft Habermas an seine Friedenspreisrede an. Dabei beruht seine Grundannahme auf der praktischen Vernunft eines nachmetaphysischen, säkularen Denkens. Vor diesem Hintergrund stützt er sich in seiner Theorie auf zwei Prämissen: Erstens geht es ihm darum zu zeigen, warum der demokratische Prozess als ein Verfahren legitimer Rechtssetzung gilt, und zweitens, warum sich Demokratie und Menschenrechte im Prozess der Verfassungsgebung gleichursprünglich miteinander verschränken (2005a: 19).

Zum ersten Punkt: Nach Habermas stellt das demokratische Verfahren der Rechtserzeugung, bei dem juristische Entscheidungsverfahren institutionalisiert und für moralische Diskurse durchlässig werden, die einzige nachmetaphysische Quelle der Legitimität dar. Dies ermögliche eine freie Diskussion von Themen, Informationen und Argumentationen, womit der politischen Willensbildung ein diskursiver Charakter zukomme und die auf diese Weise zustande gekommenen Resultate als mehr oder weniger vernünftig gelten könnten. Wenn ,die Legitimität des grundsätzlich änderbaren Zwangsrechts als Verfahrensrationalität begriffen und letztlich auf ein geeignetes kommunikatives Arrangement für die vernünftige politische Willensbildung des Gesetzgebers (und für die Rechtsanwendung) zurückgeführt wird, braucht das unverfügbare Moment der Rechtsgeltung nicht in blindem Dezisionismus zu verschwinden, noch muss es durch eine eindämmende Moral vor dem Sog der Temporalität bewahrt werden“ (Habermas 1998c: 668). Damit wird die Legitimität der Legalität nicht durch bestimmte Inhalte selbst bestimmt, sondern durch Verfahren, „die Begründungsforderungen und den Weg zu ihrer argumentativen Einlösung institutionalisieren“" (Habermas 1998b: 563).

Zum zweiten Punkt: Hier konstatiert Habermas (1999: 298 f.) eine „Gleichursprünglichkeit" von Demokratie und Menschenrechten. Einerseits dürften Menschenrechte - so moralisch sie auch zu begründen seien - nicht einem Souverän ,,paternalistisch übergestülpt" werden. Letztlich sollen sich die Bürger als die Adressaten des Rechts zugleich auch als dessen Autoren verstehen können. Andererseits könne es ohne Grund- bzw. Freiheitsrechte, die die private Autonomie der Bürger sichern, auch kein Medium für die rechtliche Institutionalisierung jener Bedingungen geben, unter denen die Bürger von ihren poli- 
tischen Teilhaberechten Gebrauch machen können. Im Rahmen eines diskursiven demokratischen Verfahrens würden sich - so Habermas - private und öffentliche Autonomie wechselseitig voraussetzen, ohne dass den Menschenrechten vor der Volkssouveränität oder umgekehrt eine Priorität zukäme. ${ }^{1}$

Im Weiteren geht Habermas - in Abgrenzung zu Böckenförde - davon aus, dass die Verfassung des liberalen Staates ihren Legitimationsbedarf „selbstgenügsam“ (2005a: 22), das heißt unabhängig von jeglichem religiösen und metaphysischen Begründungsaufwand, bestreiten könne. Zugleich ist ihm bewusst, dass jede Demokratie von politischen Tugenden abhängig ist, die sich nur in einer Zivilgesellschaft bilden können - und diese lebt von „vorpolitischen Quellen“ (2005a: 23). Doch auch hier verweist er auf den demokratischen Prozess selbst, der als das „einigende Band“ (2005a: 24) innerhalb der Gesellschaft verstanden wird. Entscheidend sei die ,gemeinsam auszuübende kommunikative Praxis“ (2005a: 24). Dass die Bürger das in gemeinschaftlichem Bemühen könnten, hätte mit der Eingewöhnung in demokratische Praktiken zu tun. Nach Habermas sind politische Tugenden ,eine Sache der Sozialisation und der Eingewöhnung in die Praktiken und Denkweisen einer freiheitlichen politischen Kultur"“ (2005a: 23).

Auch wenn der demokratische Verfassungsstaat aus Habermas' Sicht keine ihm innewohnenden Schwächen aufweist, könnten dennoch externe Gründe nicht ausgeschlossen werden, die die notwendige Solidarität auszehren, auf die der demokratische Staat angewiesen ist. Dann würde genau jene Konstellation eintreten, die Böckenförde im Blick hatte. Derartige Gefahren sieht Habermas mit einer „entgleisenden Modernisierung“ aufkommen, mit einer politisch unbeherrschten Dynamik von Weltwirtschaft und Weltgesellschaft, aber auch angesichts der Bioethik und eines zunehmend zu beobachtenden szientistischen Naturalismus. Ob die ambivalente Moderne sich allein aus säkularen Kräften einer kommunikativen Vernunft stabilisieren wird, bleibe letztlich aber eine „offene empirische Frage“ (Habermas 2005a: 28).

Vor diesem Hintergrund dürfe sich die säkularisierte Gesellschaft trotz der Trennung von Politik und Religion nicht der normativen Gehalte religiöser Überlieferung verschließen. Der liberale Staat, der mit einer politischen Verallgemeinerung einer säkularistischen Weltsicht unvereinbar ist, müsse ein Interesse an der Freigabe religiöser Stimmen in der politischen Öffentlichkeit haben (Habermas 2005a: 36, 2005b: 137). Da die Religion Bedeutungspotenziale besitze, die in profaner Rede erst noch zu erschließen seien, fordert Habermas - in Anlehnung an seine Friedenspreisrede - einen zweifachen und komplementären Lernprozess (2005a: 31 f.). Sein Ziel ist es, die religiösen Gehalte für den öffentlichen Diskurs zu übersetzen. Dabei betrachtet er die Übersetzungsarbeit als ,eine kooperative Aufgabe“ (2005b: 137), an der sich auch nicht-religiöse Bürger zu beteiligen hätten. Säkularisierte Staatsbürger dürften ,weder religiösen Weltbildern grundsätzlich ein Wahrheitspotenzial absprechen, noch den gläubigen Mitbürgern das Recht bestreiten, in religiöser Sprache Beiträge zu öffentlichen Diskussionen zu machen. Eine liberale politische Kultur kann sogar von den säkularisierten Bürgern erwarten, dass sie sich an Anstrengungen beteiligen, relevante Beiträge aus der religiösen in eine öffentlich zugängliche Sprache zu

1 Habermas 1998a: 129 ff., 1998c: 669 ff., 1999: $298 \mathrm{ff}$. 
übersetzen“ (2005a: 36). Auf diese Weise solle vermieden werden, dass sich die säkulare Gesellschaft „,von wichtigen Ressourcen der Sinnstiftung“" abschneide (2001: 22).

Im Gegensatz zu früheren Arbeiten misst Habermas der Religion nun eine gesellschaftlich relevante Bedeutung bei. Zum einen sollen sich religiöse Bürger aktiv am politischen Willensbildungsprozess beteiligen, zum anderen soll aber auch die säkulare Gesellschaft ein aktives Interesse für die Artikulation religiöser Stimmen in der politischen Öffentlichkeit entwickeln. Dabei hat Habermas bei der vorgenommenen Weiterentwicklung seiner Religionsphilosophie ${ }^{2}$ vor allem die Optimierung des öffentlichen Diskursprozesses im Blick.

\section{b) Joseph Ratzinger}

Während Habermas' Grundannahmen auf einem nach wie vor nachmetaphysischen Denken beruhen, geht Ratzinger - wie zu erwarten - metaphysisch von der jeder rationalen gemeinschaftlichen Festsetzung vorausliegenden Wirklichkeit des Menschen als Geschöpf seines Schöpfers aus. Ein laikaler Staat müsse sich ,,auf die prägenden moralischen Wurzeln stützen, die ihn gebaut haben; er darf und muss die grundlegenden Werte anerkennen, ohne die er nicht geworden wäre und ohne die er nicht überleben kann. Ein Staat der abstrakten, geschichtslosen Vernunft kann nicht bestehen.“ (2005b: 137) Hier findet er sich in völliger Übereinstimmung mit Böckenförde.

Als Vertreter der Kirche besitzt er eine naturrechtliche Perspektive auf die Welt, wonach die Natur als das sittliche Vorbild gilt, in dem sich das Wirken des Schöpfergottes offenbare. Dabei gehe Gott der Natur voraus; er gebe der Natur nicht nur Grund, sondern auch Bestand (Ratzinger 2000: I; Horster 2006: 52). Das Naturrecht sei - so Ratzinger - in der katholischen Kirche auch die Argumentationsfigur geblieben, mit der sie in Gesprächen mit der säkularen Gesellschaft an die gemeinsame Vernunft appelliere und die Grundlagen für eine Verständigung über die ethischen Prinzipien des Rechts in der säkularen pluralistischen Gesellschaft suche (2005a: 50). In diesem naturrechtlichen Sinne gebe es auch „stehende Werte, die aus dem Wesen des Menschseins folgen und daher für alle Inhaber dieses Wesens unantastbar sind“ (2005a: 44).

Die von Habermas prognostizierten Gefahren aufgreifend beschreibt er sowohl gegenwärtige Pathologien in der Religion in Form von Terror, der sich aus religiösen Quellen speist, als auch Pathologien der Vernunft wie die Existenz der Atombombe oder Gefahren der Bioethik (Ratzinger 2005a: 46 f.). Dabei habe der Prozess der Begegnung und Durchdringung der Kulturen, aber auch die Veränderung des Welt- und Menschenbildes durch die Wissenschaft zum Zerbrechen moralischer Gewissheiten beigetragen (2005a: 40 f.).

Ein originäres Problem sieht Ratzinger im Auseinanderdriften von Glaube und Vernunft. In diesem Zusammenhang bestehe auch für das Christentum die Gefahr, seine historische Errungenschaft der Vereinigung von ratio und religio - das heißt der Erkenntnis

2 Dabei handelt es sich nicht um eine Religionsphilosophie im eigentlichen Sinne. Habermas geht es nicht um religiöse Inhalte selbst, sondern um die Rolle, die Religion in der Gesellschaft spielen kann. So betrachtet er Religion von außen und weist ihr von dieser Außenperspektive einen eigenen und spezifischen Stellenwert zu. 
als Realität des Göttlichen und des Kultes als politisches Phänomen, vom Staat zu dessen Verehrung und zum rechten Verhalten der Bürger geschaffen - wieder zu verlieren. So sei eine generelle Skepsis gegenüber dem Wahrheitsanspruch der Religion durch die Wissenschaft erkennbar. Ratzinger konstatiert: „Durch die Evolutionstheorie scheint die Schöpfungslehre überholt; durch die Erkenntnisse über den Ursprung des Menschen die Erbsündenlehre; die kritische Exegese relativiert die Gestalt Jesu und setzt Fragezeichen gegenüber seinem Sohnesbewusstsein; der Ursprung der Kirche in Jesus erscheint zweifelhaft und so fort. Die philosophische Grundlage des Christentums ist durch das Ende der Metaphysik problematisch geworden, seine historischen Grundlagen stehen infolge der modernen historischen Methoden im Zwielicht. So liegt es auch von daher nahe, die christlichen Inhalte ins Symbolische zurückzunehmen, ihnen keine höhere Wahrheit zuzusprechen als den Mythen der Religionsgeschichte" (Ratzinger 2005c: 132). Vor diesem Hintergrund beklagt er einen Relativismus in seiner eigenen Kirche, dem man entgegenwirken müsse.

Abschließend konstatiert er die „,faktische Nichtuniversalität der beiden großen Kulturen des Westens, der Kultur des christlichen Glaubens wie derjenigen der säkularen Rationalität“" (2005a: 54), da auch die Evidenz der säkularen Rationalität an bestimmte kulturelle Kontexte gebunden sei und an ihre Grenzen stoße. Letztlich gebe es weder einen rationalen noch einen ethischen oder religiösen weltumfassenden Konsens.

Vor diesem Hintergrund spricht er von einer notwendigen Korrelationalität von Vernunft und Religion bzw. Vernunft und Glaube und erhebt beide zur wechselseitigen Kontrollinstanz. Hier greift er auf die Enzyklika FIDES ET RATIO zurück, die vom „Drama der Trennung zwischen Glaube und Vernunft" spricht. So habe die Vernunft ohne den Beitrag der Offenbarung Seitenwege eingeschlagen und sich in die Gefahr begeben, ihr letztes Ziel aus dem Blick zu verlieren. Der Glaube wiederum, dem die Vernunft fehle, habe Empfindung und Erfahrung betont und stehe damit in Gefahr, kein universales Angebot mehr darstellen zu können (Papst Johannes Paul II 1998: Pkt. 48). Nach Ratzinger bedarf die Vernunft der Religion zum Schutz vor Reduktionismus. Ein entleerter, säkularer Vernunftbegriff trage dazu bei, dass sich der Mensch selbst verkürze, da die eigentlich menschlichen Fragen nach dem Woher und Wohin außen vor blieben. Haben aber Fragen der Religion keinen Platz mehr im Bereich der Wissenschaft und Vernunft, würden sie ins Subjektive verlegt, womit die Religion ihre gemeinschaftsbildende Kraft verliere und der Beliebigkeit verfalle. Glaube und Vernunft könnten nur wieder zueinander finden, wenn die selbstverfügte Beschränkung der Vernunft auf das im Experiment Falsifizierbare überwunden und der Vernunft ihre ganze Weite wieder eröffnet werde (Benedikt XVI 2006: 29 f.). Andererseits bedürfe aber auch die Religion der Vernunft, um diese vor irrationalen und fundamentalistischen Tendenzen zu bewahren.

Die beiden Hauptpartner in dieser Korrelationalität seien - so Ratzinger (2005a: 57) der christliche Glaube und die westliche säkulare Rationalität. Dabei hätten beide aber auch auf die Stimmen der anderen Kulturen zu hören. In diesem Kontext ist gleichzeitig Ratzingers Verständnis eines gewissermaßen hierarchisch geordneten religiösen Pluralismus zu betrachten. In der jüngsten und viel beachteten Regensburger Vorlesung von Benedikt XVI. zu Glaube und Vernunft findet sich dies explizit ausgeführt. So bestehe ein enger 
Zusammenhang zwischen biblischer Botschaft und griechischem Denken, eine innere Analogie zwischen Gott und Vernunft: „Nicht vernunftgemäß handeln ist dem Wesen Gottes zuwider" (Benedikt XVI. 2006: 16 f.). Dementsprechend sei für das Christentum die Einheit von Glaube und Vernunft konstitutiv. Allerdings konstatiert Benedikt XVI. auch innerhalb des Christentums drei Enthellenisierungswellen, die zum Auseinanderdriften von Glaube und Vernunft beigetragen hätten: die Reformation, die liberale Theologie des 19. und 20. Jahrhunderts sowie das derzeitige Aufkommen von Religionsgemeinschaften (wie beispielsweise der Pfingstkirchen in Lateinamerika), die sich zwar auf die Botschaft des Neuen Testaments berufen, nicht aber die Synthese mit dem Griechentum vollziehen. Damit ist für Joseph Ratzinger bzw. Benedikt XVI. vor allem der Protestantismus für das Auseinanderbrechen von Glaube und Vernunft innerhalb des Christentums verantwortlich - ein essenzieller Grund, diesem auch den Status der Kirche abzusprechen.

Gleichfalls grenzt er sich von anderen Religionen wie dem Islam ab. So sei für die moslemische Lehre Gott absolut transzendent und sein Wille an keine Kategorie - auch nicht an die der Vernünftigkeit - gebunden. Mithin unterscheidet er zwischen dem „Willkür-Gott" des Islam und dem dreieinigen Gott des Christentums als dem liebenden Vernunftwesen. Auf diese Weise nimmt er jeweils prägnante und harte Abgrenzungen mit klar erkennbaren kultur- und religionspolitischen Intentionen vor (Graf 2006: 16).

\section{Vergleich beider Ansätze}

Nach dieser skizzierten Gegenüberstellung der Hauptargumentationslinien beider Protagonisten bleibt die Frage nach Übereinstimmungen, Divergenzen und Komplementaritäten der vorgestellten Ansätze. Beginnend mit der sich deckenden Analyse der gegenwärtigen Gefahren einer entgleisenden Modernisierung reflektieren beide - Habermas und Ratzinger - die Frage, ob angesichts dieser Bedingungen der säkulare Verfassungsstaat seine normativen Bestandsvoraussetzungen aus eigenen Ressourcen erneuern könne. Ausgehend von diametralen Grundpositionen kommen beide Dialogpartner über sehr unterschiedliche Ansätze zu gleichen Schlussfolgerungen. Während Habermas die Religion aus der Perspektive der irrtumsanfälligen Freiheit einer ambivalenten Moderne betrachtet, sieht Ratzinger, überzeugt vom Wahrheitsanspruch der Religion, skeptisch auf die eingeschlagenen Wege der säkularen Vernunft. Vor diesem Hintergrund sind beide von der Notwendigkeit eines wechselseitigen Wahrnehmungs- und Lernprozesses überzeugt. Dementsprechend konnte Joseph Ratzinger am Ende des Dialogs auch feststellen: „Hinsichtlich der praktischen Konsequenzen finde ich mich in weitgehender Übereinstimmung mit dem, was Jürgen Habermas über eine postsäkulare Gesellschaft, über die Lernbereitschaft und die Selbstbegrenzung nach beiden Seiten hin ausgeführt hat"“ (2005a: 56).

Die Ausgangspunkte und Wege, die zu dieser Schlussfolgerung führten, sind dagegen sehr verschieden. Zunächst teilen zwar beide Protagonisten die Genesis-Hypothese und sehen in bestimmten Entwicklungen im Bereich der Religion - speziell des Christentums eine wesentliche Voraussetzung der historischen Genese von Demokratie in Europa. So seien auch nach Habermas (1988: 23) Begriffe wie Moralität und Sittlichkeit, Person und 
Individualität, Freiheit und Emanzipation ohne jüdisch-christliches Denken nicht verstehbar. Hinsichtlich der Geltungshypothese, das heißt inwieweit die Demokratie beständig auf Religion angewiesen ist, gehen indessen die Sichtweisen auseinander. Für den Nachmetaphysiker Habermas sind die rechtsstaatliche Demokratie und säkulare Vernunft gemäß der von ihm entwickelten Diskurstheorie prinzipiell durchaus in der Lage, ihre Normativität aus sich selbst zu schöpfen. Inwieweit eine ambivalente Moderne konkret dazu in der Lage ist, lässt er indessen als eine offene empirische Frage stehen, womit eine gewisse Unsicherheit unverkennbar scheint. Dagegen argumentiert Ratzinger aus einem metaphysischen Denken. So könne der Mensch ohne Religion, einen absoluten Bezug und die Unverfügbarkeit Gottes nicht die Unbedingtheit und Universalität moralischer Prinzipien begründen. Vor diesem Hintergrund geben beide Protagonisten auch unterschiedliche Antworten auf das Böckenförde-Diktum. Dabei unterscheidet Habermas zwei Aggregatzustände und differenziert zwischen einem Stabilisierungsmechanismus, bei dem das System in gewisser Weise idealtypisch ohne Störungen funktioniert, und einem, der jedwede externe Störung mit einbezieht. Nur für diesen letztgenannten Fall lässt er empirisch offen, ob die Demokratie auf externe Voraussetzungen angewiesen ist. Nach Ratzinger bedarf der liberale demokratische Staat zu seiner Stabilisierung stets der Religion.

Eine Schlüsselposition nimmt dabei der Begriff der Vernunft ein. Beide Denker gebrauchen diesen in unterschiedlicher Weise und Bedeutung. Ratzinger besitzt ein transzendentes, auf Gott bezogenes Verständnis von Vernunft. So sei der Mensch als Geschöpf Gottes - entsprechend der Analogielehre - durch Gott mit seiner Vernunft begabt. Angesichts dessen fordert Ratzinger auch die Einheit von Glaube und Vernunft. Demgegenüber geht Habermas von einem säkularen Vernunftbegriff aus. Er fragt nach den Erkenntnisbedingungen. Dabei bewegt er sich in der Tradition Kants. Allerdings sieht er diese keineswegs metaphysisch begründet oder postuliert. Vernunft entfaltet sich nach Habermas in der zwischenmenschlichen Kommunikation, insbesondere in Form von Sprache. In diesem Lichte ist auch sein Diskursprinzip zu sehen.

Ein weiterer gravierender Unterschied zwischen Habermas und Ratzinger ergibt sich aus der unterschiedlichen - philosophischen respektive theologischen - Sichtweise auf die Religion (Horster 2006: 52 f.). Wenn Habermas den religiösen Bürgern im säkularen Staat eine Stimme in eigener Klangfarbe gewähren will, dann ist die Religion für ihn nur ein Teilsystem der Gesellschaft, denn den Anspruch auf das Interpretationsmonopol und eine umfassende Lebensgestaltung musste die Religion „unter Bedingungen der Säkularisierung des Wissens, der Neutralisierung der Staatsgewalt und der verallgemeinerten Religionsfreiheit aufgeben. Mit der funktionalen Ausdifferenzierung gesellschaftlicher Teilsysteme trennt sich auch das Leben der religiösen Gemeinde von ihren sozialen Umgebungen.“ (Habermas 2005a: 34) Demgegenüber ist Ratzinger von der Religion als umfassende Lebensgestaltung und -orientierung überzeugt. Er spricht sich für eine umfassende Prägung des Menschen und seines Lebensentwurfs durch die Religion und eine Orientierung des Menschen an der Religion aus (2004: 22). Gleichzeitig muss er allerdings erkennen - und darin trifft sich Ratzinger wieder mit Habermas - dass das Christentum nicht mehr die Lebensform einer ganzen Gesellschaft sei oder in absehbarer Zeit werden könne (Ratzinger 2004: 175). Angesichts dessen spricht er auch von der Nichtuniversalität 
der beiden großen Kulturen des Westens: des christlichen Glaubens und der säkularen Rationalität (2005a: 54).

\section{Kritik}

Der von Habermas und Ratzinger geführte Dialog hat hinsichtlich der Frage nach den normativen Bestandsvoraussetzungen des säkularen demokratischen Verfassungsstaates bzw. der Frage, inwieweit und ob überhaupt die Religion zur Stabilisierung der Demokratie nutzbar gemacht werden kann, im Grunde zu keinen neuen Erkenntnissen geführt.

Beide Protagonisten gehen von unterschiedlichen Perspektiven aus, die zwar komplementär aufeinander verweisen, aber letztlich keine Lösung aufzeigen können. Einerseits kann Ratzinger argumentativ genau die Lücke füllen, die Habermas offen lässt - die Frage der Verankerung moralischen Verhaltens im Lichte einer ambivalenten Moderne. Andererseits fehlt dem Christentum aber die dafür erforderliche Universalität, was wiederum auf die zunehmende Notwendigkeit eines gesellschaftlichen Diskurses - eines Dialogs mit der säkularen Gesellschaft sowie den anderen Kulturen - verweist, sodass ,ein universaler Prozess der Reinigungen wachsen kann, in dem letztlich die von allen Menschen irgendwie gekannten oder geahnten wesentlichen Werte und Normen neue Leuchtkraft gewinnen können, so dass wieder zu wirksamer Kraft in der Menschheit kommen kann, was die Welt zusammenhält"“ (Ratzinger 2005a: 57 f.). Dabei lässt Ratzinger völlig offen, in welcher Weise dies geschehen kann, denn einen Konsens hält er selbst für nicht gegeben, ein Weltethos sei bloße Abstraktion (2005a: 55). Letztlich findet seine Argumentation ihre Grenze sowohl im religiösen Pluralismus als auch im säkularen Staat. Religion kann nur über die Bürger selbst, die - jedenfalls in Europa - soziologisch betrachtet schon weitgehend säkularisiert sind, öffentlich wirksam werden. Auch sein Verweis auf Habermas und die Frage eines Teheraner Kollegen, ob nicht aus kulturvergleichender und religionssoziologischer Sicht die europäische Säkularisierung der Sonderweg sei, der einer Korrektur bedürfe, verkennt die Unumkehrbarkeit der Geschichte. In Europa richtete sich die Trennung von Staat und Kirche gegen den politischen Herrschaftsanspruch der Kirchen; es ging um eine Begrenzung der Macht der Kirchen. In den USA handelte es sich dagegen um ein Trennungsmodell zum Selbstschutz religiöser Minderheiten. Dort wurde der Staat in seine Schranken verwiesen.

Im Fokus des Habermas'schen Ansatzes steht die Übersetzung religiöser Semantik in eine öffentlich zugängliche Sprache, damit sich die säkulare Gesellschaft nicht von wichtigen Ressourcen der Sinnstiftung abschneide. Angesichts der religiösen Herkunft der moralischen Grundlagen des liberalen Staates räumt er der religiösen Sprache - wenn auch konjunktivisch formuliert - einen uneinholbaren Artikulationsvorsprung ein (Habermas 2001: 23). Mit dem Korrektiv der Religion auf die Herausforderung der entgleisenden Moderne, die sich in der „Sprache des Marktes“ ausdrückt, wird die religiöse Semantik bei ihm zugleich in den Zusammenhang der These von der Notwendigkeit eines lebensweltlichen Aufbegehrens gegen die expansive Dynamik systemischer Imperative und ihrer Medien gestellt. Bedenkt man, dass genau darin - in der Übernahme einer ,Ausfallbürgschaft" für die desintegrativen Folgen der sozialen Differenzierung - für Habermas der 
eigentliche Sinn des politischen Systems liegt, wird der demokratietheoretische Stellenwert dieser Aussage deutlich (Habermas 1998a: 366; Haus 2003: 55).

In diesem Zusammenhang bleibt die Frage offen, weshalb es religiöser Botschaften bedarf und wodurch Religion in der Lage ist, den Sinn von Humanität zu begründen. Hier sieht Habermas die Grenze der Philosophie in der „Irreversibilität vergangenen Leidens“ (2001: 24). So hinterlasse „die verlorene Hoffnung auf Resurrektion [...] eine spürbare Leere“ (2001: 24 f.). Daraus folgert er, dass ,semantische Potentiale noch nicht ausgeschöpft“ (2001: 25) seien. In diesem Kontext ist die von ihm vorgeschlagene Übersetzungsarbeit zu werten, die in der Habermas'schen Diskurstheorie zugleich ein neues Stück Verfahrensrationalität darstellt und auf diesem Wege auch zu einer Aufwertung der Religion in der Öffentlichkeit führt.

Letztlich bleibt aber zu fragen, inwieweit semantische Potenziale ausgeschöpft werden können, wenn Religion - so wie Habermas es tut - ausschließlich aus der Außenperspektive betrachtet wird. Zum einen könnte man eine prinzipielle Unübersetzbarkeit der Glaubenswahrheiten ins Feld führen, wenn Glaube als ein ,kognitiver Habitus“ (Habermas 2005c: 229) betrachtet wird, der nicht über äußerliche Bildung, sondern nur über Sozialisation erreichbar ist. Zum anderen - so die Hauptkritik am Habermas'schen Ansatz - scheint unabhängig davon jede postsäkulare Übersetzung hinsichtlich bleibender Desiderate unvollkommen. Eine kommunikative, postsäkulare Rationalität kann den Begriff des Absoluten, die Unverfügbarkeit Gottes, nicht aufheben, ohne dass dabei unweigerlich Bedeutungspotenziale verloren gehen. Das sieht auch Habermas, entsprechend konstatiert er: „Ein Nullsummenspiel ist schon deshalb ausgeschlossen, weil die ,intellektualistische“ Erwartung, religiöse Erfahrungsgehalte, die in der Kult- und Gebetspraxis einer Gemeinde verwurzelt sind, ließen sich ohne Rest in Inhalte einer öffentlichen Diskurs- und Begründungspraxis überführen, abwegig ist" (Habermas 2007a: 400). Insbesondere lassen sich die transzendenten - weil nicht im öffentlichen Diskurs erschließbaren - Elemente der Religion und die transzendente Verankerung moralischen Verhaltens nicht übersetzen. Mit einer Profanisierung des religiösen Erbes müssen sich letztlich auch die moralischen Sensibilitäten, die einst religiös eingeübt wurden, verlieren, denn gerade diese transzendente Verankerung sorgte dafür, dass entsprechende Handlungsregeln, Wertmaßstäbe und Sinnvorstellungen befolgt wurden. Damit ist ein entscheidender Funktionsgewinn im Hinblick auf die Stabilität der Demokratie durch eine bloße Übersetzung religiöser Semantik in eine öffentlich zugängliche Sprache nicht zu erwarten. Es bleibt spannend, wie Jürgen Habermas seine Religionsphilosophie weiterentwickeln wird, ohne sein Diskursprinzip zu verwerfen.

Der Dialog zwischen Habermas und Ratzinger hat, aufbauend auf der Habermas'schen Friedenspreisrede, eine breite Debatte, nicht nur um die vorpolitischen Grundlagen des demokratischen Verfassungsstaates, sondern gleichfalls um das Unbestimmbare in der Demokratie, die Frage nach der Existenz einer postsäkularen Gesellschaft und das Verhältnis von Glauben und Wissen in der Öffentlichkeit entfacht. So haben unter anderem in den Jahren 2004 und 2005 zwei internationale Symposien in Wien zum Thema Glauben und Wissen (Langthaler/Nagl-Docekal 2007) stattgefunden, bei denen die jüngsten Stellungnahmen Habermas' zur Religion im Mittelpunkt des Interesses standen. Im Februar 2007 
richtete eine hoch besetzte internationale Konferenz in Bochum in Anlehnung an den in diesem Aufsatz betrachteten Dialog zwischen Habermas und Ratzinger ihr Interesse auf aktuelle Fragen der Dialektik der Säkularisierung. ${ }^{3}$ Dort wurde gleichfalls die zentrale Frage diskutiert, was eine Gesellschaft noch zusammenhält, nachdem Religion, zumindest in Westeuropa, diese Funktion immer mehr verliert.

Aber auch die beiden Protagonisten - Jürgen Habermas und Papst Benedikt XVI. führen die Gedanken ihres Dialogs weiter. In diesem Kontext stieß insbesondere die bereits angesprochene Regensburger Rede des Papstes Benedikt XVI. über Glaube und Vernunft vom Dezember 2006 auf eine breite Resonanz. Nur zwei Monate später, im Februar 2007, hat Habermas in der Neuen Züricher Zeitung (2007b) dazu öffentlich Stellung bezogen: Der Papst habe seiner jüngsten Regensburger Rede eine ,unerwartet modernitätskritische Wendung“ (2007b) gegeben, indem er zwar die Auffassung kritisiert habe, „man müsse nun wieder hinter die Aufklärung zurückgehen und die Einsichten der Moderne verabschieden“ (Benedikt XVI. 2006: 29), sich aber gegen die Kraft der Argumente gestemmt habe, an denen jene weltanschauliche Synthese zerbrochen sei.

Zugleich hat Habermas in seiner Replik auf die Regensburger Rede explizit auf das Motiv seiner jüngsten Beschäftigung mit dem Thema Religion und Glauben verwiesen. So geht es ihm vor allem darum, ,die moderne Vernunft gegen den Defaitismus, der in ihr selber brütet, zu mobilisieren“"(2007b). Das betrifft zum einen die fehlenden Formen einer säkularen Kontingenzbewältigung, zum anderen die schon von Kant diagnostizierte Schwäche der Vernunftmoral. Denn der Entschluss zum solidarischen Handeln im Anblick von Gefahren, die nur durch kollektive Anstrengungen gebannt werden können, sei nicht nur eine Frage der Einsicht. Dabei verfehle die praktische Vernunft ,ihre eigene Bestimmung, wenn sie nicht mehr die Kraft hat, in profanen Gemütern ein Bewusstsein für die weltweit verletzte Solidarität, ein Bewusstsein von dem, was fehlt, von dem, was zum Himmel schreit, zu wecken und wachzuhalten“ (2007b).

Bestehen bleibt die säkulare Gewissheit, dass „der Glaube für das Wissen etwas Opakes [behält], das weder verleugnet, noch bloss hingenommen werden darf" (2007b). Angesichts dessen wird die weitere Debatte darum geführt werden müssen, wie der weltanschaulich neutrale Staat einen modus vivendi zwischen einer religiösen Uniformität einerseits und einem Säkularismus andererseits, der religiöse Äußerungen für schlechthin irrational und abwegig hält, finden kann. Mit einer bloßen Religionsfreiheit kann der Staat - so auch Habermas (2007b) - nicht zufrieden sein. Denn zu seiner Legitimation bedarf er einer festen Verwurzelung seiner essenziellen Grundlagen im Bewusstsein gläubiger, andersgläubiger wie ungläubiger Bürger gleichermaßen.

\section{Literatur}

Alexy, Robert, 1990: Zur Kritik des Rechtspositivismus, in: Rechtspositivismus und Wertbezug des Rechts (Archiv für Rechts- und Sozialphilosophie, Beiheft 37), 9-26.

3 Vgl. http://www.ruhr-uni-bochum.de/Ispraktphil/Flyer.pdf (Stand: 20.04.07). 
Benedikt XVI., 2006: Glaube und Vernunft. Die Regensburger Vorlesung, Freiburg/Basel/ Wien.

Böckenförde, Ernst-Wolfgang, 2006: Die Entstehung des Staates als Vorgang der Säkularisation, in: ders., Recht, Staat, Freiheit, Frankfurt a. M. (erw. Aufl.), 92-114. (Zuerst 1967)

Forschner, Maximilian, 2002: Naturrecht, in: Otfried Höffe (Hrsg.), Lexikon der Ethik, München (6. neu bearbeitete Aufl.), 186-188.

Graf, Friedrich Wilhelm, 2006: Eine Wissenschaft, die sich für das Ganze zuständig weiß, in: SZ, 06.12.06, S. 16.

Habermas, Jürgen, 1988: Nachmetaphysisches Denken. Philosophische Aufsätze, Frankfurt a. M.

Habermas, Jürgen, 1998a: Faktizität und Geltung. Beiträge zur Diskurstheorie des Rechts und des demokratischen Rechtsstaats, Frankfurt a. M. (Zuerst 1992)

Habermas, Jürgen, 1998b: Tanner Lectures von 1986, in: ders. 1998a, 541-599. (Zuerst 1986)

Habermas, Jürgen, 1998c: Nachwort zur vierten Auflage von Faktizität und Geltung von 1994, in: ders. 1998a, 661-680. (Zuerst 1994)

Habermas, Jürgen, 1999: Die Einbeziehung des Anderen. Studien zur politischen Theorie, Frankfurt a. M. (Zuerst 1996)

Habermas, Jürgen, 2001: Glauben und Wissen. Friedenspreis des Deutschen Buchhandels 2001, Frankfurt a. M.

Habermas, Jürgen, 2005a: Vorpolitische Grundlagen des demokratischen Rechtsstaates?, in: ders./Joseph Ratzinger, Dialektik der Säkularisierung. Über Vernunft und Religion, Freiburg/Basel/Wien, 15-37.

Habermas, Jürgen, 2005b: Religion in der Öffentlichkeit. Kognitive Voraussetzungen für den öffentlichen Vernunftgebrauch religiöser und säkularer Bürger, in: ders., Zwischen Naturalismus und Religion. Philosophische Aufsätze, Frankfurt a. M., 119-154.

Habermas, Jürgen, 2005c: Die Grenze zwischen Glauben und Wissen. Zur Wirkungsgeschichte und aktuellen Bedeutung von Kants Religionsphilosophie, in: ders., Zwischen Naturalismus und Religion. Philosophische Aufsätze, Frankfurt a. M., 216-257.

Habermas, Jürgen, 2007a: Replik auf Einwände, Reaktion und Anregungen, in: Rudolf Langthaler/Herta Nagl-Docekal (Hrsg.), Glauben und Wissen. Ein Symposium mit Jürgen Habermas, Wien, 366-414.

Habermas, Jürgen, 2007b: Ein Bewusstsein von dem, was fehlt. Über Glauben und Wissen und den Defaitismus der modernen Vernunft, in: Neue Züricher Zeitung vom 10.02.07, http://www.nzz.ch/2007/02/10/li/articleEVB7X.print.html (Stand: 20.04.07).

Hoerster, Norbert, 1990: Zur Verteidigung der rechtspositivistischen Trennungsthese, in: Rechtspositivismus und Wertbezug des Rechts (Archiv für Rechts- und Sozialphilosophie, Beiheft 37), 27-32.

Horster, Detlef, 2006: Jürgen Habermas und der Papst. Glauben und Vernunft, Gerechtigkeit und Nächstenliebe im säkularen Staat, Bielefeld.

Kelsen, Hans, 1928: Die philosophischen Grundlagen der Naturrechtslehre und des Rechtspositivismus, Berlin.

Kelsen, Hans, 1960: Reine Rechtslehre, Wien (2. Aufl.). 
Langthaler, Rudolf/Nagl-Docekal, Herta, 2007: Glauben und Wissen. Ein Symposium mit Jürgen Habermas, Wien.

Mann, Thomas, 2004: Joseph und seine Brüder. Der vierte Roman: Joseph, der Ernährer, Frankfurt a. M. (12. Aufl.).

Münkler, Herfried, 1993: Zivilgesellschaft und Bürgertugend. Bedürfen demokratisch verfasste Gemeinwesen einer sozio-moralischen Fundierung?, Berlin.

Münkler, Herfried, 1994: Subsidiarität, Zivilgesellschaft und Bürgertugend, in: Alois Riklin/ Gerard Batliner (Hrsg.), Subsidiarität, Baden-Baden, 63-80.

Münkler, Herfried, 1996: Einleitung: Was sind vorpolitische Grundlagen politischer Ordnung?, in: ders. (Hrsg.), Bürgerreligion und Bürgertugend. Debatten über die vorpolitischen Grundlagen politischer Ordnung, Baden-Baden, 7-11.

Papst Johannes Paul II, 1998: Enzyklika FIDES ET RATIO. Glaube und Vernunft, Stein am Rhein.

Ratzinger, Joseph, 2000: Der angezweifelte Wahrheitsanspruch. Die Krise des Christentums am Beginn des dritten Jahrtausends, in: FAZ, 08.01.00, S. I-II.

Ratzinger, Joseph, 2004: Salz der Erde. Christentum und katholische Kirche im neuen Jahrtausend. Ein Gespräch mit Peter Seewald, München (5. Aufl.).

Ratzinger, Joseph, 2005a: Was die Welt zusammenhält. Vorpolitische moralische Grundlagen eines freiheitlichen Staates, in: Jürgen Habermas/ders., Dialektik der Säkularisierung. Über Vernunft und Religion, Freiburg/Basel/Wien, 39-60.

Ratzinger, Joseph, 2005b: Werte in Zeiten des Umbruchs. Die Herausforderungen der Zukunft, Freiburg/Basel/Wien.

Ratzinger Joseph, 2005c: Glaube - Wahrheit - Toleranz. Das Christentum und die Weltreligionen, Freiburg/Basel/Wien.

Schuller, Florian, 2005: Vorwort, in: Jürgen Habermas/Joseph Ratzinger, Dialektik der Säkularisierung. Über Vernunft und Religion, Freiburg/Basel/Wien, 7-14.

Seibert, Christoph, 2006: Inklusion von Religion im politischen Diskurs. Eine irreführende Fragestellung? (unveröffentlichtes Vortragsmanuskript).

Korrespondenzanschrift:

PD Dr. Ines-Jacqueline Werkner

12555 Berlin

Kaulsdorfer Str. 135

E-Mail: werkner@zedat.fu-berlin.de 